\title{
INTEGRAL OPERATORS IN BANACH SPACES
}

\author{
J. R. HOLUB
}

ABstract. Giving a new proof of a result of Grothendieck, it is shown that a weak operator limit of a bounded net of integral maps is again integral and that every integral map is a weak operator limit of a net of finite dimensional maps which are uniformly bounded in nuclear norm. It is also shown that the subnuclear maps defined by Ruckle are precisely the integral maps. Using this result some questions of Ruckle are answered.

1. In a recent paper [6] Ruckle has introduced a class of operators called subnuclear operators and asked whether every such operator is nuclear. In this paper we show that the subnuclear maps are precisely the integral maps of Grothendieck [3] and give a characterization of integral maps as weak operator limits of bounded nets of nuclear maps.

2. Definitions and preliminary results. Let $E$ and $F$ be Banach spaces and $\mathscr{L}(E, F)$ the space of all bounded linear operators from $E$ to $F$. Throughout the paper the term "operator" or "map" will mean $a$ bounded linear transformation.

We will denote by $E \otimes_{\epsilon} F$ the completion of the algebraic tensor product $E \otimes F$ in the $\epsilon$-norm defined by

$$
\left\|\sum_{i=1}^{n} x_{i} \otimes y_{i}\right\|_{\epsilon}=\sup _{\|f\|_{\lessgtr 1, f \in E^{*} ;\left\|_{g}\right\|_{\lessgtr 1, g} \in F^{*}}}\left|\sum_{i=1}^{n}\left\langle f, x_{i}\right\rangle\left\langle g, y_{i}\right\rangle\right| .
$$

Similarly, we denote by $E \otimes_{\pi} F$ the completion of $E \otimes F$ in the $\pi$ norm defined by

$$
\left\|\sum_{i=1}^{n} x_{i} \otimes y_{i}\right\|_{\pi}=\inf \sum_{j=1}^{k}\left\|x_{j}^{\prime}\right\|\left\|y_{j}^{\prime}\right\|,
$$

where the infimum is taken over all

$$
\sum_{j=1}^{k} x_{j}^{\prime} \otimes y_{j}^{\prime}=\sum_{i=1}^{n} x_{i} \otimes y_{i} .
$$

An operator $T: E \rightarrow F$ is called nuclear $[3$, p. 80] if there exist sequences $\left(f_{i}\right) \subset E^{*}$ and $\left(y_{i}\right) \subset F$ for which $\sum_{i}\left\|f_{i}\right\|\left\|y_{i}\right\|<+\infty$ and

Received by the editors July 31, 1970.

AMS 1970 subject classifications. Primary 47B10; Secondary 46B99.

Key words and phrases. Integral operator, subnuclear operator, nuclear operator. weak operator topology. 
$T x=\sum_{i}\left\langle f_{i}, x\right\rangle y_{i}$ for all $x \in E$. The nuclear norm $\gamma(T)$ of a nuclear operator $T: E \rightarrow F$ is the infimum of all sums $\sum_{i}\left\|f_{i}\right\|\left\|y_{i}\right\|$ where $T$ has the representation $T x=\sum_{i=1}^{\infty}\left\langle f_{i}, x\right\rangle y_{i}$ as above. The space of all nuclear operators from $E$ to $F$ under the nuclear norm will be denoted by $N(E, F)$. Whenever we refer to a "bounded" set of nuclear operators we always will mean "bounded in nuclear norm." It is well known and easy to see that every element $\sum_{i=1}^{\infty} f_{i} \otimes y_{i}$ of $E^{*} \otimes_{\pi} F$ determines a nuclear map $T$ from $E$ to $F$ defined by $T(x)=\sum_{i} f_{i}(x) y_{i}$.

The space $\left(E \otimes{ }_{6} F^{*}\right)^{*}$ has been characterized by Grothendieck as the space of all integral bilinear forms on $E \times F^{*}\left[3\right.$, p. 124]. If $T_{B}$ is a bilinear form on $E \times F^{*}$ then it induces in a natural way a linear form, call it $q$, on $E \otimes F^{*}$ defined by $\langle q, x \otimes g\rangle=T_{B}(x, g)$. If $q$ is continuous in the $\epsilon$-topology on $E \otimes F^{*}$ then $T_{B}$ is said to be an integral bilinear form (the term "integral" is derived from Grothendieck's characterization of such forms as integrals over products of equicontinuous subsets of $E^{*}$ and $\left.F^{* *}\right)$. If $T \in \mathcal{L}(E, F)$ then $T$ induces a bilinear form $T_{B}$ on $E \times F^{*}$ according to the formula $T_{B}(x, g)=\langle T x, g\rangle$ for $x \in E$, $g \in F^{*}$. If the induced bilinear form $T_{B}$ is integral then the operator $T$ is said to be an integral operator. The integral norm of such an operator $T$ is denoted by $I(T)$ and is defined to be the norm of the linear functional $q$ in $\left(E \otimes_{\epsilon} F^{*}\right)^{*}$ which it determines as above. The space of all integral maps from $E$ to $F$ will be denoted by $I(E, F)$. Whenever we refer to a "bounded" set of integral operators we mean "bounded in integral norm." It is well known that every nuclear map is integral and, surprisingly, the composition of an integral map and a weakly compact map is nuclear [3, p. 133]. Recent results in the theory of integral operators [10] indicate the important role these mappings play in the isomorphic characterization of Banach spaces.

\section{A characterization of integral operators.}

(3.1) Theorem. Let $T: E \rightarrow F$ be such that there is a bounded net $\left(T_{\alpha}\right)$ of integral maps from $E$ to $F$ which converges in the weak operator topology to $T$. Then $T$ is an integral operator.

Proof. By assumption there is a constant $M>0$ such that

(i) $\sup _{\alpha} I\left(T_{\alpha}\right) \leqq M$, and

(ii) $T_{\alpha}(x)(g) \rightarrow T(x)(g)$ for all $x \in E, g \in F^{*}$.

According to the remarks in $\$ 2$ let $q$ and $q_{\alpha}$ denote the linear forms on $E \otimes_{\epsilon} F^{*}$ corresponding to the operators $T$ and $T_{\alpha}$ respectively. By (i) above, $\sup _{\alpha}\left\|q_{\alpha}\right\| \leqq M$. We show that $q$ is continuous on $E \otimes_{\mathrm{C}} F^{*}$.

Let $\sum_{i=1}^{n} x_{i} \otimes g_{i} \in E \otimes{ }_{\epsilon} F^{*}$. Then by (ii) above, given $\delta>0$ there is an $\alpha$ such that 
That is,

$$
\left|\sum_{i=1}^{n} T\left(x_{i}\right)\left(g_{i}\right)\right| \leqq\left|\sum_{i=1}^{n} T_{\alpha}\left(x_{i}\right)\left(g_{i}\right)\right|+\delta
$$

$$
\left|q\left(\sum_{i=1}^{n} x_{i} \otimes g_{i}\right)\right| \leqq\left|q_{\alpha}\left(\sum_{i=1}^{n} x_{i} \otimes g_{i}\right)\right|+\delta \leqq M\left\|\sum_{i=1}^{n} x_{i} \otimes g_{i}\right\|_{e}+\delta .
$$

Since $\delta>0$ was arbitrary,

$$
\left|q\left(\sum_{i=1}^{n} x_{i} \otimes g_{i}\right)\right| \leqq M\left\|\sum_{i=1}^{n} x_{i} \otimes g_{i}\right\|_{\epsilon}
$$

and $q$ is continuous on $E \otimes_{\epsilon} F^{*}$. By definition $T$ is integral.

(3.2) Corollary. Let $T: E \rightarrow F^{*}$ be such that there is a bounded net of integral operators from $E$ to $F^{*}$ for which $T_{\alpha}(x)(y) \rightarrow T(x)(y)$ for all $x \in E$ and $y \in F$. Then $T$ is an integral operator.

Proof. As the argument of (3.1) shows, the operator $T$ defines a linear form $q$ on $E \otimes_{\epsilon} F$ which is continuous. Thus by a theorem of Grothendieck [3, p. 128] $T$ is integral.

(3.3) CoRollary. If $T: E \rightarrow F$ is a weak operator limit of a bounded net of nuclear operators from $E$ to $F$ then $T$ is integral.

Proof. It is well known that every nuclear operator $S$ is integral and $I(S) \leqq \gamma(S)$ [3, p. 127]. The corollary now follows from (3.1).

Our next result shows that the property expressed in (3.3) actually characterizes integral operators.

(3.4) TheOREM. Let $T \in \mathfrak{L}(E, F)$ be an integral map. Then there exists a bounded net $\left(T_{\alpha}\right)$ in $N(E, F)$ such that $\left(T_{\alpha}\right)$ converges to $T$ in the weak operator topology.

Proof. According to results of Schatten the injection map $j: E \otimes_{\mathrm{e}} F^{*} \rightarrow E^{* *} \otimes_{\mathrm{c}} F^{*} \rightarrow\left(E^{*} \otimes_{\pi} F\right)^{*}$ is an isometry [8]. Thus the adjoint map $j^{*}:\left(E^{*} \otimes_{\pi} F\right)^{* *} \rightarrow\left(E \otimes_{\epsilon} F^{*}\right)^{*}$ is a topological homomorphism onto $\left(E \otimes_{\epsilon} F^{*}\right)^{*}$.

If $T: E \rightarrow F$ is integral then the associated linear form $q$ on $E \otimes F^{*}$ defined by $\langle q, x \otimes g\rangle=T x(g)$ is continuous and hence contained in $\left(E \otimes_{\epsilon} F^{*}\right)^{*}$.

Since $j^{*}$ is onto $\left(E \otimes_{\mathrm{C}} F^{*}\right)^{*}$ there is then an $S \in\left(E^{*} \otimes_{\pi} F\right)^{* *}$ such that $\|S\| \leqq\|q\|+1$ and $\langle S, y\rangle=\langle q, y\rangle$ for all $y \in E \otimes_{\epsilon} F^{*}$ (recall that the mapping $j^{*}$ is just the restriction map).

By Goldstine's theorem [8, p. 143] there exists a net $\left(T_{\alpha}\right)$ in $E^{*} \otimes_{\pi} F$ such that $\sup _{\alpha}\left\|T_{\alpha}\right\| \leqq\|S\|<+\infty$ and $\left\langle T_{\alpha}, z\right\rangle \rightarrow\langle S, z\rangle$ for all 
$z \in\left(E^{*} \otimes_{\pi} F\right)^{*}$. Hence certainly $\left\langle T_{\alpha}, y\right\rangle \rightarrow\langle S, y\rangle$ for all $y \in E \otimes_{\epsilon} F^{*}$, and in particular for $y=x \otimes g$ where $x \in E, g \in F^{*}$. Thus if $x \in E, g \in F^{*}$, $T_{\alpha} x(g)=\left\langle T_{\alpha}, x \otimes g\right\rangle \rightarrow\langle S, x \otimes g\rangle=\langle q, x \otimes g\rangle$. But $\langle q, x \otimes g\rangle=T x(g)$, so $T_{\alpha} x(g) \rightarrow T x(g)$. Since the net $\left(T_{\alpha}\right)$ is bounded in $N(E, F)$ the theorem is proved.

(3.5) REMARK. In general one cannot replace "net" by "sequence" in the statement of Theorem (3.4).

To see this, let $i: L^{\infty}(\mu) \rightarrow L^{1}(\mu)$ be the (integral, [3, p. 128]) injection map for a measure space $(S, \Sigma, \mu)$ in which $i\left(L^{\infty}(\mu)\right)$ is a nonseparable linear subspace of $L^{1}(\mu)$. If there exists a sequence $\left(T_{n}\right)$ of nuclear operators such that $T_{n} f(g) \rightarrow i(f)(g)$ for all $f, g \in L^{\infty}(\mu)$, then since every nuclear operator is compact and therefore has separable range, we would have that $U_{n} T_{n}\left(L^{\infty}(\mu)\right)$ is separable. Thus the convex hull $C$ of $U_{n} T_{n}\left(L^{\infty}(\mu)\right)$ is also separable. Since $C$ is convex, its weak and norm closures in $L^{1}(\mu)$ coincide $[8$, p. 65] and hence the weak closure of $C$, call it $\bar{C}^{\omega}$, is separable.

Thus there must be an $f \in L^{\infty}(\mu)$ such that $i(f) \notin \bar{C}^{\omega}$ (since $i\left(L^{\infty}(\mu)\right)$ is nonseparable in $\left.L^{1}(\mu)\right)$. But then $\left\langle T_{n} f, g\right\rangle$ could not converge to $\langle i(f), g\rangle$ for all $g \in L^{\infty}(\mu)$, a contradiction to our original assumption.

(3.6) Remark. As our proof of (3.4) shows, the mappings $\left(T_{\alpha}\right)$ may all be chosen to be finite dimensional since the finite dimensional maps are dense in $N(E, F)$.

Combining (3.3) and (3.4) we get the following characterization of integral maps.

(3.7) Theorem. A mapping $T: E \rightarrow F$ is integral if and only if $T$ is the limit in the weak operator topology on $\mathcal{L}(E, F)$ of a bounded net of nuclear operators from $E$ to $F$.

4. Integral and subnuclear operators. If $E$ is a Banach space with a basis then a mapping $T: E^{*} \rightarrow E^{*}$ is called subnuclear [6] if there exists a bounded sequence $\left(T_{k}\right)$ in $N\left(E^{*}, E^{*}\right)$ such that $T_{k}(f)(x)$ $\rightarrow T(f)(x)$ for all $f \in E^{*}$ and $x \in E$. As we mentioned in the introduction, Ruckle has introduced the concept of a subnuclear map and asked whether every subnuclear map is nuclear. Our purpose in this section is to show that the classes of subnuclear and integral maps on $E^{*}$ coincide and therefore every subnuclear map on $E^{*}$ is nuclear only if every integral map is nuclear.

(4.1) Theorem. Let $E$ be a Banach space with a basis $\left(x_{i}, f_{i}\right)$. Then a mapping $T: E^{*} \rightarrow E^{*}$ is subnuclear if and only if $T$ is integral.

Proof. If $T$ is subnuclear then, by Corollary (3.2), $T$ is integral. 
Conversely, suppose $T$ is integral. Then $T^{*}: E^{* *} \rightarrow E^{* *}$ is also integral (this is well known, e.g. [3], but also follows easily from (3.3) and (3.4)). In the remainder of the proof we will identify $E$ with its canonical image in $E^{* *}$ and $E^{*}$ with its image in $E^{* * *}$.

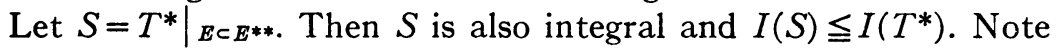
that $S: E \rightarrow E^{* *}$ and $S^{*} f=T f$ for all $f \in E^{*} \subset E^{* * *}$. Now since $\left(x_{i}, f_{i}\right)$ is a basis for $E$ there is a sequence of projections $P_{n}: E \rightarrow E$ defined by

$$
P_{n}(x)=\sum_{i=1}^{n}\left\langle f_{i}, x\right\rangle x_{i}
$$

for which $\sup _{n}\left\|P_{n}\right\|<+\infty$.

Define $S_{n}: E \rightarrow E^{* *}$ by $S_{n}=S \circ P_{n}$. Then if $Q_{n}=\left.S\right|_{\left[x_{i}\right]_{i-1}^{n}}$, each $Q_{n}$ is nuclear (being finite dimensional) and since the dual of $\left[x_{i}\right]_{i=1}^{n}$ has the metric approximation property it follows from a result of Grothendieck that the nuclear and integral norms of $Q_{n}$ are the same for all $n\left[3\right.$, p. 180]; i.e. $N\left(Q_{n}\right)=I\left(Q_{n}\right)$. Since $Q_{n}$ is the restriction of $S$ to a subspace of $E, I\left(Q_{n}\right) \leqq I(S)<+\infty$ and therefore

$$
\sup _{n} N\left(Q_{n}\right)=\sup _{n} I\left(Q_{n}\right)<+\infty \text {. }
$$

It is clear that $S_{n}=Q_{n} \circ P_{n}$. Hence

$$
\sup _{n} N\left(S_{n}\right) \leqq\left[\sup _{n} N\left(Q_{n}\right)\right]\left[\sup _{n}\left\|P_{n}\right\|\right]<+\infty \quad[3] \text {. }
$$

Moreover since $S_{n}(x) \stackrel{n}{\rightarrow} S(x)$ for all $x \in E$ we have $\left\langle S_{n} x, f\right\rangle^{\stackrel{n}{\rightarrow}}\langle S x, f\rangle$ for all $x \in E, f \in E^{*}$.

Now $S_{n}^{*}: E^{* * *} \rightarrow E^{*}$ is nuclear for each $n$ and $\sup _{n} N\left(S_{n}^{*}\right)$ $\leqq \sup N\left(S_{n}\right)<+\infty[3$, p. 85$]$. Thus the sequence $\left(T_{n}\right)$ in $\mathcal{L}\left(E^{*}, E^{*}\right)$ defined by

$$
T_{n}=\left.S_{n}^{*}\right|_{E^{*} \subset E^{* * *}}
$$

is a bounded sequence of nuclear operators on $E^{*}$. We show $\left\langle T_{n} f, x\right\rangle$ $\stackrel{n}{\rightarrow}\langle T f, x\rangle$ for all $f \in E^{*}$ and $x \in E$.

To see this, note that $\left\langle T_{n} f, x\right\rangle=\left\langle S_{n}^{*} f, x\right\rangle=\left\langle f, S_{n}(x)\right\rangle$ for all $f \in E^{*}$, $x \in E$, and all $n$. According to our earlier remarks, $\left\langle f, S_{n} x\right\rangle \stackrel{n}{\rightarrow}\langle f, S x\rangle$ $=\left\langle S^{*} f, x\right\rangle$. But this last is just $\langle T f, x\rangle$.

Hence by definition $T$ is subnuclear.

It is now easy to construct of subnuclear map which is not nuclear. To obtain such an example let $i: L^{\infty}[0,1] \rightarrow L^{1}[0,1]$ be the injection map. It is well known that $i$ is integral $[3$, p. 128], but clearly it is not compact. Let $S: L^{1}[0,1] \rightarrow L^{\infty}[0,1]$ be an isometric embedding of the 
separable space $L^{1}[0,1]$ into the space $L^{\infty}[0,1]$ (which is universal for all separable spaces [4]). Then $T=S \circ i$ is an integral map on $L^{\infty}[0,1]=\left(L^{1}[0,1]\right) *[3$, p. 128$]$ which is not compact and hence not nuclear $[3$, p. 82$]$. Since $L^{1}[0,1]$ has a basis (e.g. the Haar functions) it follows from Theorem 4.1 that $T$ is subnuclear and is therefore the desired example.

(4.2) REMARK. The proof of Theorem 4.1 shows that if $T: E \rightarrow F$ is integral and $E$ has a basis, then $T$ is a weak operator limit of a bounded sequence of nuclear operators. A similar (though slightly more involved) argument shows that if $F$ has a basis the same conclusion holds. The assumption that $E$ or $F$ has a basis is crucial, as the example in (3.5) shows.

\section{REFERENCES}

1. M. M. Day, Normed linear spaces, Ergebnisse der Mathematik und ihrer Grenzgebiete, Heft 21, Springer-Verlag, Berlin, 1958. MR 20 \#1187.

2. N. Dunford and J. Schwartz, Linear operators. I: General theory, Pure and Appl. Math., vol. 7, Interscience, New York, 1958. MR 22 \#8302.

3. A. Grothendieck, Produits tensoriels topologiques et espaces nucléaires, Mem. Amer. Math. Soc. No. 16 (1955). MR 17, 763.

4. J. Lindenstrauss and A. Pełczyński, Absolutely summing operators in $L_{p}$-spaces and their applications, Studia Math. 29 (1968), 275-326. MR 37 \#6743.

5. A. Pietsch, Nukleare lokalkonvexe Räume, Schriftenreihe bei der Deutschen Akad. Wissenschaften Berlin. Reihe A, Reine Mathematik, Heft 1, Akademie-Verlag, Berlin, 1965. MR 31 \#6114.

6. W. Ruckle, Decompositions of operator spaces, Rev. Roumaine Math. Pures Appl. 15 (1970), 119-134.

7. H. Schaefer, Topological vector spaces, Macmillan, New York, 1966. MR 33 \#1689.

8. R. Schatten, $A$ theory of cross spaces, Ann. of Math. Studies, no. 26, Princeton Univ. Press, Princeton, N. J., 1950. MR 12, 186.

9. L. Schwartz, Séminaire Schwartz Année 1953/54, Secrétariat mathématique, Paris, 1954. MR 17, 764.

10. C. P. Stegall and J. R. Retherford, Fully nuclear and completely nuclear operators with applications to $\mathfrak{L}_{\mathbf{1}}$ and $\mathfrak{L}_{\infty}$ spaces, Trans. Amer. Math. Soc. (to appear).

Virginia Polytechnic Institute and State University, Blacksburg, VirGINIA 24061 